\title{
The Effect Of Auditor Change On Initial Audit Fee Discount And Non-Audit Services
}

\author{
Hong-jo Park, Daegu University, South Korea \\ Jeong-un Choi, Daegu University, South Korea \\ Joonhei Cheung, Daegu University, South Korea
}

\begin{abstract}
The purpose of this research is to verify whether non-audit services are provided without additional fees at the initial audit as a strategy to win an external audit contract, which could give the appearance of initial audit fee discount. From the results, non-audit services are provided at the initial audit, and the initial audit fee is discounted accordingly, only when the independent auditor is changed from a Big 4 accounting firm to a non-Big 4 accounting firm. However, there is no meaningful relevance in any other types of changes. Therefore, if the auditor is changed from a Big 4 accounting firm to a non-Big 4 accounting firm, non-audit services are provided without additional fees in order to win an external audit contract, and the publication of audit fees with a division between the audit service fee and the non-audit service fee may give the appearance of a discounted audit service fee.
\end{abstract}

Keywords: Initial Audit; Audit Fee Discount; Non-Audit Services; Auditor Change

\section{INTRODUCTION}

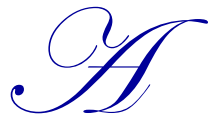

$\mathrm{n}$ independent auditor generally provides audit service and non-audit services simultaneously if the audit is continuously maintained. For an independent auditor, maintaining a close and long-term relationship with an auditee is essential in providing a profitable non-audit service. This tendency is empirically verified by Beck et al. (1988a), who showed that a company purchasing repetitive non-audit services such as taxation, pension or information system from an independent auditor has a longer audit service period by the same auditor.

However, more auditors provide an audit service and non-audit services simultaneously after being appointed as an independent auditor, even though it is initial audit. Although not considered a general matter, certain exceptional factors may apply. In this research, we focus on the provision of non-audit services without additional fees by an accounting firm in order to win an external audit contract. Therefore, a new auditor is more likely to receive a level of total fees similar to those paid to the predecessor, while also providing additional non-audit services which were not provided by the predecessor. Moreover, although additional fees are not charged for these non-audit services provided, the officially reported audit fees are lower than those of the previous auditor because of the published division of audit fees and non-audit fees, which can be misrepresented as an initial audit fee discount.

The purpose of this research is to verify whether accounting firms provide non-audit services without additional fees at the initial audit as a strategy to win an audit contract, which could give the appearance of a discounted initial audit fee. According to previous research, this tendency is expected to occur when the independent auditor is changed to a small auditor. Therefore, whether the relevance between an audit fee discount at the initial audit and the provision of non-audit services is dependent on the type of auditor change is verified through an empirical analysis. 


\section{LITERATURE REVIEW AND HYPOTHESES}

\section{Literature Review}

DeAngelo (1981) suggested the theoretical possibility of an initial audit fee discount based on the start-up cost of the initial audit and the transactional costs of an auditor change. This indicates that the start-up cost of a new auditor and the auditor conversion cost of the auditee, which are generated at auditor change, allow the current auditor to use quasi-rents, and that the auditor trying to acquire a new contract may suggest an initial audit fee discount based on predictable future quasi-rents. Many previous studies suggested such an initial audit fee discount phenomenon (Francis and Simon 1987; Simon and Francis 1988; Walker and Casterella 2000; Ghosh and Lustgarten 2006; Desir et al. 2014).

However, Dye (1991) approached audit fees from the perspective of information, and thus argued that the initial audit fee discount is not a transactional cost suggested by DeAngelo (1981), but it is based on the assumption of the bargaining power of the auditor in the process of deciding the audit fee. In other words, he expected that if the company under audit has all of bargaining power in the process of deciding audit fees, no initial audit fee discount will be offered since there are no future rents for the auditor. Therefore, if audit fees are published, the effect of future quasirents is decreased, and no more initial audit fee discount will be offered. Subsequently, Craswell and Francis (1999) presented an empirical result based on data in Australia showing that no initial audit fee discount is offered except in the case of escalation change from non-Big8 to Big8.

Studies on non-audit services have focused on the independence issue of the auditor through simultaneous provision of audit and non-audit services. Since the simultaneous provision of audit service and non-audit services might disrupt the independence of the auditor due to the close relationship between the auditor and the company under audit, the Securities and Exchange Commission (SEC) has required the publication of non-audit services from 2001. In relation to this, Dee et al. (2002) suggested that the accrual is increased as the portion of non-audit fees increases. In particular, Frankel et al. (2002) presented a negative association between the portion of non-audit services fee at the publication of audit fees and the stock returns.

However, even the portion of fees for non-audit services has consistently increased. Antle et al. (2006) proposed a negative association between non-audit services fee and abnormal accrual based on the knowledge transfer phenomenon due to the simultaneous provision of non-audit services. Moreover, DeFond et al. (2002) proposed that there is no meaningful relation between the portion of non-audit services fee and the auditor's opinion on the uncertainty for going-concern business, and argued that this is based on the auditor's reputation.

\section{Hypotheses}

In consideration of the knowledge transfer phenomenon due to simultaneous provision of audit and non-audit services mostly presented by the previous research related to non-audit services, the simultaneous and repetitive provision of non-audit services is considered possible in a continuous audit. The non-audit services that are provided based on information of the company under audit collected from an audit increases profits. Therefore, the non-audit services are provided more frequently during the continuous audit period than the initial audit year.

However, in consideration of the fact that the initial audit fee discount arises due to the competition between auditors to win an audit contract, a different supposition can be postulated. In the case of a large auditor (BIG), which has a relative cost advantage due to its size (or technical efficiency based on industrial expertise), the initial audit fee discount may be large due to the quasi-rents during the future audit period, whereas the inferior small auditor (nonBIG) presents a limited initial audit fee discount (Ettredge and Greenberg 1990). At this time, the small auditor (nonBIG), which has a limited initial audit fee discount, will establish a strategy to win a new audit contract through other measures rather than the initial audit fee discount. In other words, it will provide the initial audit and additional nonaudit services simultaneously, which may induce the possibility to follow a non-pricing strategy rather than a pricing strategy using the initial audit fee discount, which is limited compared to that of the large auditor (BIG). Therefore, the following hypothesis is postulated. 
Hypothesis 1: (In a situation where the non-audit services are relatively unusual at the initial audit year) The initial audit where a large auditor (BIG) is changed to a small auditor (non-BIG), non-audit services will probably be offered.

The unreasonable audit fees could be a sign as the possibility of disrupting the auditor' independence to the information user. If the audit fees are below a certain level, the economic relationship based on quasi-rents for the future audit period can be assumed, and if the audit fees exceed a certain level, then an audit opinion may be purchased. At this time, in consideration of the high quality of audit from a large auditor (BIG), there is no benefit in changing to a small auditor (non-BIG) from a large auditor (BIG) at higher audit fees, so it is likely for a small auditor (non-BIG) to offer lower audit fees (DeAngelo 1981; Becker et al. 1998).

However, the non-pricing strategy to provide non-audit services simultaneously may be a practical alternative since the issue of the auditor's independence may be raised due to the significantly lower audit fees and discounts are limited due to the poor cost advantage compared to a large auditor (BIG). This strategy may avoid the independence issue by maintaining similar audit fees compared to the large auditor (BIG), while obtaining a discount effect of audit fees by providing non-audit services simultaneously. Therefore, a small auditor provides additional non-audit services similar to the total fees provided by a large auditor (BIG) to compete against the large auditor (BIG) by discounting the fees per service. Therefore, the following hypothesis is postulated.

Hypothesis 2: The initial audit fee discount in terms of non-audit services is probably offered in the case of changing to a small auditor (non-BIG) from a large auditor (BIG).

\section{SELECTION OF SAMPLE AND RESEARCH DESIGN}

\section{Selection of Sample}

We analyze the listed companies on the KOSPI and KOSDAQ from 2003 to 2014 . We collect financial data and corporate governance data from the KISVALUE database and audit fees and non-audit service fees from TS-2000. We select firms with a December 31 fiscal year-end. Lastly, we exclude firms that belong to the financial industry. All variables are winsorized at the 1st and 99th percentiles. The final sample size is 11,578 observations.

\section{Research Model}

We estimate the relation between non-audit services and auditor change using Equation (1). In order to calculate the probability of a client receiving a non-audit service, we use the results of the following logit model:

$$
\begin{aligned}
& p\left(N A S R_{D_{i t}}\right)=\alpha_{0}+\alpha_{1} B_{1} G_{N O N B I G_{i t}}+\alpha_{2} N O N B I G_{-} B I G_{i t}+\alpha_{3} B_{1} G_{-} B I G_{i t}+\alpha_{4} N O N B I G_{-} N O N B I G_{i t}+ \\
& \alpha_{5} L A S T_{i t}+\alpha_{6} S I Z E_{i t}+\alpha_{7} L E V_{i t}+\alpha_{8} G R W_{i t}+\alpha_{9} M B_{i t}+\alpha_{10} R O A_{i t}+\alpha_{11} C F O_{i t}+\alpha_{12} O W N E R_{i t}+ \\
& \alpha_{13} F_{O R G} I_{i t}+\alpha_{14} B_{I G} 4_{i t}+\alpha_{15} K_{O S P I}+I N D+Y D+e
\end{aligned}
$$

\begin{tabular}{|c|c|}
\hline$N A S F \_D:$ & 1 if the client receives non-audit services for auditor and 0 otherwise; \\
\hline$B I G \_N O N B I G$ : & 1 if changes a large auditor to a small auditor and 0 otherwise; \\
\hline$N O N B I G \_B I G$ : & 1 if changes a small auditor to a large auditor and 0 otherwise; \\
\hline$B I G \_B I G:$ & 1 if changes a large auditor to a large auditor and 0 otherwise; \\
\hline $\begin{array}{l}\text { NONBIG_NONBIG: } \\
\text { LAST: }\end{array}$ & 1 if changes a small auditor to a small auditor and 0 otherwise; \\
\hline $\begin{array}{l}\text { LASI: } \\
\text { SIZE: }\end{array}$ & $\begin{array}{l}1 \text { if last audit and } 0 \text { otherwise; } \\
\text { The natural logarithm of total assets; }\end{array}$ \\
\hline$L E V:$ & Total liabilities divided by total assets; \\
\hline$G R W:$ & Sales divided by beginning sales; \\
\hline$M B:$ & Market value divided by book value; \\
\hline$R O A:$ & Net income divided by lagged total assets; \\
\hline CFO: & Operating cash flow divided by total assets; \\
\hline OWNER: & Ownership of largest shareholder and related parties; \\
\hline FORGI: & Foreign ownership; \\
\hline BIG4: & 1 if auditor is a large auditor called Big 4 and 0 otherwise; \\
\hline KOSPI: & 1 if firms are listed in KOSPI and 0 otherwise; \\
\hline
\end{tabular}




$\begin{array}{ll}\text { IND: } & \text { industry dummy } \\ Y D: & \text { year dummy }\end{array}$

Our variables of interest are BIG_NONBIG, NONBIG_BIG, BIG_BIG and NONBIG_NONBIG. We expect that the probability of a client receiving a non-audit services are more pronounced for firms changing a large auditor to a small auditor. Therefore, we predict $\alpha 1$ to be positive or not significant but $\alpha 2, \alpha 3, \alpha 4$ to be negative in support of $\mathrm{H} 1$. We include control variables (LAST, SIZE, LEV, GRW, MB, ROA, CFO, OWNER, FORGI, BIG4, KOSPI) as in Kim et al. (2016).

We estimate the relation the initial audit fee discount in terms of non-audit services and types of auditor change using Equation (2):

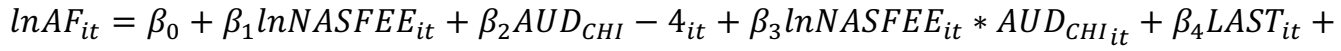

$$
\begin{aligned}
& \beta_{5} R O A_{i t-1}+\beta_{6} L_{O S S_{i t-1}}+\beta_{7} L E V_{i t-1}+\beta_{8} \text { SIZE }_{i t-1}+\beta_{9} G R W_{i t-1}+\beta_{10} \text { EXPRATIO }_{i t-1}+ \\
& \beta_{11} L I Q_{i t-1}+\beta_{12} I_{N V A R_{i t-1}}+\beta_{13} M B_{i t-1}+\beta_{14} \text { OWNER } R_{i t}+\beta_{15} \text { FORGI }_{i t}
\end{aligned}
$$

$\ln A F: \quad$ The natural logarithm of audit fee;

InNASFEE: $\quad$ The natural logarithm of non-audit fee;

AUD_CH1-4;

(1) $B \bar{I} G \_N O N B I G$ : $\quad 1$ if changes a large auditor to a small auditor and 0 otherwise;

(2) $N O \bar{N} B I G \_B I G$ : 1 if changes a small auditor to a large auditor and 0 otherwise;

(3) $B I G \_B I G$ : $\quad 1$ if changes a large auditor to a large auditor and 0 otherwise;

(4) NONBIG_NONBIG: 1 if changes a small auditor to a small auditor and 0 otherwise;

LOSS:

EXPRATIO:

$L I Q:$

INVAR:

OPINO:

1 if a firm reports loss and 0 otherwise;

international sales divided by sales;

current asset divided current liability

Receivables and inventory divided by total assets;

1 if audit opinion is clean and 0 otherwise;

Our variable of interest is lnNASFEEit*AUD_CH. Then we perform a regression analysis using Equation (2) across subsamples of firms with audit change type (AUD_CH1-4). The variables AUD_CH1-4 are BIG_NONBIG, NONBIG_BIG, BIG_BIG and NONBIG_NONBIG. We only expect a negative coefficient of lnNASFEE*A-AUD_CH1 (BIG_NONBIG) in line with Hypothesis 2 . The control variable LART, ROA, LOSS, LEV is a proxy for audit risk and SIZE, GRW, EXPRATO are a proxy for client size (Simunic 1980; Francis 1984). We also include liquidity control variables (LIQ and INVAR) and corporate governance control variables (OWNER, FORGI). Simunic (1980) and Francis (1984) demonstrated that audit fees are positively associated with auditor's disqualified opinion, so we include audit opinion. We control for Book value to Market value (MB), because Book value to Market value captures various factors to audit fees in growing firms. Lastly, for all specifications of the research model, we include industry and year dummy to control for industry and year effects.

\section{EMPIRICAL RESULTS}

\section{Descriptive Statistics and Correlation Analysis}

Table 1 provides descriptive statistics for the variables included in Equations (1) and (2) for the full sample. The mean NASF_D is $28.95 \%$ and the mean of lnNASFEE is 4.8669 . The mean (median) of lnAF is 18.0058 (17.9099). FIRST is $15.40 \%$. The mean of the interesting variables BIG_NONBIG is $3.21 \%$.

Table 2, Panel A shows the Pearson correlation among the variables used in Equation (1) and Panel B shows the Pearson correlation among the variables used in Equation (2). The variable NASF_D shows a significant negative correlation at $1 \%$ significance level with the variable BIG_NONBIG. This result reveals a negative relation between non-audit services and changing from a large auditor to a small auditor. The lnAF and NONBIG_BIG variables show a significant negative correlation, which means that audit fees are negatively associated with firm changes from a large auditor to a small auditor. 
Table 1. Descriptive Statistics

\begin{tabular}{|c|c|c|c|c|c|c|c|}
\hline Variables & Mean & Std. & Min & 1 st & Median & $3^{\text {rd }}$ & Max \\
\hline$N A S F \_D$ & 0.2895 & 0.4536 & 0.0000 & 0.0000 & 0.0000 & 1.0000 & 1.0000 \\
\hline $\ln N A S F E E$ & 4.8669 & 7.6628 & 0.0000 & 0.0000 & 0.0000 & 15.4249 & 19.9054 \\
\hline $\ln A F$ & 18.0058 & 0.6417 & 16.8112 & 17.5958 & 17.9099 & 18.2582 & 20.2124 \\
\hline FIRST & 0.1540 & 0.3610 & 0.0000 & 0.0000 & 0.0000 & 0.0000 & 1.0000 \\
\hline$L A S T$ & 0.1536 & 0.3605 & 0.0000 & 0.0000 & 0.0000 & 0.0000 & 1.0000 \\
\hline BIG_NONBIG & 0.0321 & 0.1764 & 0.0000 & 0.0000 & 0.0000 & 0.0000 & 1.0000 \\
\hline$N O \overline{N B} B I G \_B I G$ & 0.0279 & 0.1647 & 0.0000 & 0.0000 & 0.0000 & 0.0000 & 1.0000 \\
\hline$B I G \_B I G$ & 0.0478 & 0.2135 & 0.0000 & 0.0000 & 0.0000 & 0.0000 & 1.0000 \\
\hline$N O \overline{N B I G \_N O N B I G}$ & 0.0461 & 0.2098 & 0.0000 & 0.0000 & 0.0000 & 0.0000 & 1.0000 \\
\hline SIZE & 25.6612 & 1.3582 & 23.2805 & 24.7398 & 25.3954 & 26.3203 & 30.2485 \\
\hline$L E V$ & 0.4086 & 0.1963 & 0.0404 & 0.2518 & 0.4081 & 0.5553 & 0.8779 \\
\hline$G R W$ & 1.1049 & 0.3572 & 0.3342 & 0.9380 & 1.0590 & 1.1968 & 2.9226 \\
\hline$M B$ & 1.3333 & 1.2299 & 0.1848 & 0.5976 & 0.9500 & 1.5937 & 7.7098 \\
\hline$R O A$ & 0.0122 & 0.1140 & -0.5681 & -0.0007 & 0.0308 & 0.0678 & 0.2128 \\
\hline $\mathrm{CFO}$ & 0.0496 & 0.1008 & -0.2641 & -0.0043 & 0.0472 & 0.1034 & 0.3469 \\
\hline OWNER & 0.2693 & 0.1382 & 0.0474 & 0.1655 & 0.2422 & 0.3482 & 0.6999 \\
\hline FOR & 0.0633 & 0.1123 & 0.0000 & 0.0014 & 0.0114 & 0.0692 & 0.5613 \\
\hline BIG4 & 0.5449 & 0.4980 & 0.0000 & 0.0000 & 1.0000 & 1.0000 & 1.0000 \\
\hline KOSPI & 0.4222 & 0.4939 & 0.0000 & 0.0000 & 0.0000 & 1.0000 & 1.0000 \\
\hline$L A G \_R O A$ & 0.0177 & 0.1133 & -0.5504 & 0.0030 & 0.0342 & 0.0739 & 0.2235 \\
\hline$L A G_{-} L O S S$ & 0.2345 & 0.4237 & 0.0000 & 0.0000 & 0.0000 & 0.0000 & 1.0000 \\
\hline$L A G_{-} S I Z E$ & 25.5854 & 1.3616 & 23.2381 & 24.6572 & 25.3218 & 26.2473 & 30.1817 \\
\hline$L A G_{-} L E V$ & 0.4071 & 0.1952 & 0.0433 & 0.2517 & 0.4057 & 0.5520 & 0.8751 \\
\hline$L A G \_G R W$ & 1.1296 & 0.4001 & 0.3405 & 0.9483 & 1.0704 & 1.2147 & 3.3131 \\
\hline$L A G \_E X P R A T I O$ & 0.2699 & 0.3054 & 0.0000 & 0.0001 & 0.1285 & 0.5005 & 0.9865 \\
\hline$L A G_{-} L I Q$ & 2.5703 & 2.9318 & 0.2541 & 1.0514 & 1.5931 & 2.7697 & 19.2912 \\
\hline$L A G I I N V A R$ & 0.2782 & 0.1541 & 0.0000 & 0.1633 & 0.2670 & 0.3811 & 0.6785 \\
\hline$L A G_{-}-M B$ & 1.3051 & 1.1964 & 0.1757 & 0.5806 & 0.9343 & 1.5808 & 7.4121 \\
\hline$L A G \_O P I N O$ & 0.9977 & 0.0482 & 0.0000 & 1.0000 & 1.0000 & 1.0000 & 1.0000 \\
\hline
\end{tabular}

Table 2. Correlation Matrix

Panel A. Correlation Matrix for Model 1

\begin{tabular}{|c|c|c|c|c|c|c|c|c|}
\hline & V1 & $\mathbf{V 2}$ & V3 & V4 & V5 & V6 & V7 & V8 \\
\hline$N A S F \_D(V 1)$ & 1.000 & & & & & & & \\
\hline$B I G \_\bar{N} O N B I G(V 2)$ & -0.032 & 1.000 & & & & & & \\
\hline$N O \overline{N B I G \_B I G(V 3)}$ & -0.003 & -0.031 & 1.000 & & & & & \\
\hline$B I G \_B I G(V 4)$ & 0.023 & -0.041 & -0.038 & 1.000 & & & & \\
\hline$N O N B I G \_N O N B I G(V 5)$ & -0.060 & -0.040 & -0.037 & -0.049 & 1.000 & & & \\
\hline $\operatorname{LAST}(V 6)$ & -0.038 & -0.078 & -0.072 & -0.095 & -0.094 & 1.000 & & \\
\hline $\operatorname{SIZE}(V 7)$ & 0.192 & -0.068 & -0.010 & 0.142 & -0.111 & -0.038 & 1.000 & \\
\hline$L E V(V 8)$ & -0.007 & -0.020 & 0.006 & 0.024 & -0.002 & 0.040 & 0.201 & 1.000 \\
\hline$G R W(V 9)$ & 0.005 & 0.003 & 0.025 & 0.003 & 0.022 & -0.009 & -0.023 & 0.045 \\
\hline$M B(V 10)$ & 0.044 & -0.008 & 0.000 & 0.006 & 0.003 & 0.030 & -0.096 & 0.066 \\
\hline$R O A(V 11)$ & 0.059 & -0.003 & 0.015 & 0.032 & -0.059 & -0.082 & 0.196 & -0.282 \\
\hline$C F O(V 12)$ & 0.068 & -0.017 & 0.008 & 0.021 & -0.050 & -0.035 & 0.074 & -0.184 \\
\hline OWNER(V13) & 0.023 & -0.015 & 0.014 & 0.025 & -0.025 & -0.020 & 0.045 & -0.021 \\
\hline$F O R(V 14)$ & 0.168 & -0.053 & -0.016 & 0.083 & -0.077 & -0.028 & 0.463 & -0.103 \\
\hline$B I G 4(V 15)$ & 0.171 & -0.199 & 0.155 & 0.205 & -0.241 & -0.024 & 0.364 & 0.031 \\
\hline KOSPI (V16) & 0.121 & -0.056 & 0.000 & 0.101 & -0.058 & 0.000 & 0.552 & 0.103 \\
\hline
\end{tabular}

(Table 1, Panel A continued on next page) 
$\underline{\text { Panel A. continued }}$

\begin{tabular}{|c|c|c|c|c|c|c|c|c|}
\hline & V9 & V10 & V11 & V12 & V13 & V14 & V15 & V16 \\
\hline$G R W(V 9)$ & 1.000 & & & & & & & \\
\hline$M B(V 10)$ & 0.102 & 1.000 & & & & & & \\
\hline$R O A(V 11)$ & 0.188 & -0.096 & 1.000 & & & & & \\
\hline $\mathrm{CFO}(\mathrm{V12})$ & 0.089 & 0.028 & 0.449 & 1.000 & & & & \\
\hline OWNER(V13) & -0.010 & -0.062 & 0.131 & 0.071 & 1.000 & & & \\
\hline$F O R(V 14)$ & -0.008 & 0.110 & 0.181 & 0.155 & 0.022 & 1.000 & & \\
\hline$B I G 4(V 15)$ & -0.021 & -0.009 & 0.092 & 0.082 & 0.092 & 0.243 & 1.000 & \\
\hline KOSPI(V16) & -0.066 & -0.166 & 0.075 & -0.025 & 0.040 & 0.263 & 0.237 & 1.000 \\
\hline
\end{tabular}

Panel B. Correlation Matrix for Model 2

\begin{tabular}{|c|c|c|c|c|c|c|c|c|c|}
\hline & V1 & V2 & V3 & V4 & V5 & V6 & V7 & V8 & V9 \\
\hline $\ln A F(V 1)$ & 1.000 & & & & & & & & \\
\hline $\ln N A S F E E(V 2)$ & 0.259 & 1.000 & & & & & & & \\
\hline$B I G \_N O N B I G(V 3)$ & -0.098 & -0.036 & 1.000 & & & & & & \\
\hline$N O N B I G \_B I G(V 4)$ & -0.028 & -0.002 & -0.031 & 1.000 & & & & & \\
\hline$B I G \_B I G(V 5)$ & 0.116 & 0.025 & -0.041 & -0.038 & 1.000 & & & & \\
\hline 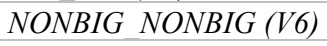 & -0.100 & -0.063 & -0.040 & -0.037 & -0.049 & 1.000 & & & \\
\hline$L A G \_R O A(V 7)$ & 0.055 & 0.055 & -0.038 & -0.005 & 0.024 & -0.069 & 1.000 & & \\
\hline$L A G \_L O S S(V 8)$ & -0.055 & -0.045 & 0.033 & -0.002 & -0.019 & 0.048 & -0.706 & 1.000 & \\
\hline$L A G \_S I Z E(V 9)$ & 0.815 & 0.222 & -0.072 & -0.013 & 0.136 & -0.113 & 0.173 & -0.155 & 1.000 \\
\hline$L A G_{-} L E V(V 10)$ & 0.239 & 0.004 & -0.014 & 0.009 & 0.021 & 0.001 & -0.288 & 0.221 & 0.221 \\
\hline$L A G \_G R W(V 11)$ & -0.021 & 0.008 & -0.005 & 0.000 & -0.012 & 0.003 & 0.191 & -0.156 & -0.018 \\
\hline$L A G \_E X P R A T I O(V 12)$ & 0.070 & 0.027 & -0.003 & -0.006 & -0.002 & -0.020 & 0.028 & 0.017 & 0.075 \\
\hline$L A G \_L I Q(V 13)$ & -0.209 & 0.007 & 0.024 & -0.011 & -0.022 & 0.017 & 0.119 & -0.072 & -0.215 \\
\hline$L A G \_I N V A R(V 4)$ & -0.168 & -0.079 & 0.013 & 0.021 & -0.043 & 0.007 & 0.091 & -0.092 & -0.168 \\
\hline$L A G \_M B(V 15)$ & 0.071 & 0.049 & 0.004 & 0.011 & 0.018 & 0.022 & -0.069 & 0.072 & -0.088 \\
\hline OWNER (V16) & -0.004 & 0.022 & -0.015 & 0.014 & 0.025 & -0.025 & 0.124 & -0.086 & 0.043 \\
\hline FOR $(V 17)$ & 0.436 & 0.189 & -0.053 & -0.016 & 0.083 & -0.077 & 0.184 & -0.149 & 0.456 \\
\hline$L A G \_O P I N O(V 18)$ & 0.000 & 0.011 & 0.009 & 0.008 & 0.002 & 0.002 & 0.027 & -0.028 & 0.013 \\
\hline
\end{tabular}

Panel B. continued

\begin{tabular}{|c|c|c|c|c|c|c|c|c|c|}
\hline & V10 & V11 & V12 & V13 & V14 & V15 & V16 & V17 & V18 \\
\hline$L A G \_L E V(V 10)$ & 1.000 & & & & & & & & \\
\hline$L A G \_G R W(V 11)$ & 0.041 & 1.000 & & & & & & & \\
\hline$L A G \_E X P R A T I O(V 12)$ & 0.049 & 0.008 & 1.000 & & & & & & \\
\hline$L A G \_L I Q(V 13)$ & -0.619 & -0.035 & -0.014 & 1.000 & & & & & \\
\hline$L A G \_I N V A R(V 4)$ & 0.188 & 0.079 & 0.029 & -0.144 & 1.000 & & & & \\
\hline$L A G_{-}^{-} M B(V 15)$ & 0.062 & 0.111 & 0.011 & 0.042 & -0.077 & 1.000 & & & \\
\hline OWNER (V16) & -0.020 & -0.013 & -0.056 & 0.035 & 0.016 & -0.059 & 1.000 & & \\
\hline FOR $(V 17)$ & -0.088 & 0.009 & 0.015 & 0.029 & -0.128 & 0.109 & 0.022 & 1.000 & \\
\hline$L A G \_O P I N O(V 18)$ & -0.022 & 0.004 & -0.006 & 0.009 & -0.005 & -0.006 & -0.010 & -0.006 & 1.000 \\
\hline
\end{tabular}

1) The lower triangle presents the Pearson correlation coefficients. Boldfaced figures are statistically significant at the 0.05 level. 
Table 2. Correlation Matrix

$\underline{\text { Panel A. Correlation Matrix for Model } 1}$

\begin{tabular}{|c|c|c|c|c|c|c|c|c|}
\hline & V1 & V2 & V3 & V4 & V5 & V6 & V7 & V8 \\
\hline$N A S F \_D(V 1)$ & 1.000 & & & & & & & \\
\hline$B I G \_\bar{N} O N B I G(V 2)$ & -0.032 & 1.000 & & & & & & \\
\hline$N O \overline{N B I G \_B I G(V 3)}$ & -0.003 & -0.031 & 1.000 & & & & & \\
\hline$B I G_{-} B I G(V 4)$ & 0.023 & -0.041 & -0.038 & 1.000 & & & & \\
\hline 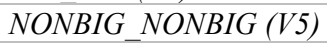 & -0.060 & -0.040 & -0.037 & -0.049 & 1.000 & & & \\
\hline $\operatorname{LAST}(V 6)$ & -0.038 & -0.078 & -0.072 & -0.095 & -0.094 & 1.000 & & \\
\hline $\operatorname{SIZE}(V 7)$ & 0.192 & -0.068 & -0.010 & 0.142 & -0.111 & -0.038 & 1.000 & \\
\hline$L E V(V 8)$ & -0.007 & -0.020 & 0.006 & 0.024 & -0.002 & 0.040 & 0.201 & 1.000 \\
\hline$G R W(V 9)$ & 0.005 & 0.003 & 0.025 & 0.003 & 0.022 & -0.009 & -0.023 & 0.045 \\
\hline$M B(V 10)$ & 0.044 & -0.008 & 0.000 & 0.006 & 0.003 & 0.030 & -0.096 & 0.066 \\
\hline$R O A(V 11)$ & 0.059 & -0.003 & 0.015 & 0.032 & -0.059 & -0.082 & 0.196 & -0.282 \\
\hline$C F O(V 12)$ & 0.068 & -0.017 & 0.008 & 0.021 & -0.050 & -0.035 & 0.074 & -0.184 \\
\hline OWNER(V13) & 0.023 & -0.015 & 0.014 & 0.025 & -0.025 & -0.020 & 0.045 & -0.021 \\
\hline FOR $(V 14)$ & 0.168 & -0.053 & -0.016 & 0.083 & -0.077 & -0.028 & 0.463 & -0.103 \\
\hline$B I G 4(V 15)$ & 0.171 & -0.199 & 0.155 & 0.205 & -0.241 & -0.024 & 0.364 & 0.031 \\
\hline KOSPI(V16) & 0.121 & -0.056 & 0.000 & 0.101 & -0.058 & 0.000 & 0.552 & 0.103 \\
\hline
\end{tabular}

Panel A. continued

\begin{tabular}{|c|c|c|c|c|c|c|c|c|}
\hline & V9 & V10 & V11 & V12 & V13 & V14 & V15 & V16 \\
\hline$G R W(V 9)$ & 1.000 & & & & & & & \\
\hline$M B(V 10)$ & 0.102 & 1.000 & & & & & & \\
\hline$R O A(V 11)$ & 0.188 & -0.096 & 1.000 & & & & & \\
\hline$C F O(V 12)$ & 0.089 & 0.028 & 0.449 & 1.000 & & & & \\
\hline OWNER(V13) & -0.010 & -0.062 & 0.131 & 0.071 & 1.000 & & & \\
\hline FOR(V14) & -0.008 & 0.110 & 0.181 & 0.155 & 0.022 & 1.000 & & \\
\hline$B I G 4(V 15)$ & -0.021 & -0.009 & 0.092 & 0.082 & 0.092 & 0.243 & 1.000 & \\
\hline KOSPI(V16) & -0.066 & -0.166 & 0.075 & -0.025 & 0.040 & 0.263 & 0.237 & 1.000 \\
\hline
\end{tabular}

Panel B. Correlation Matrix for Model 2

\begin{tabular}{|c|c|c|c|c|c|c|c|c|c|}
\hline & V1 & $\mathbf{V} 2$ & V3 & V4 & V5 & V6 & V7 & V8 & V9 \\
\hline $\ln A F(V 1)$ & 1.000 & & & & & & & & \\
\hline $\ln N A S F E E(V 2)$ & 0.259 & 1.000 & & & & & & & \\
\hline$B I G \_N O N B I G(V 3)$ & -0.098 & -0.036 & 1.000 & & & & & & \\
\hline$N O N B I G \_B I G(V 4)$ & -0.028 & -0.002 & -0.031 & 1.000 & & & & & \\
\hline$B I G \_B I G(V 5)$ & 0.116 & 0.025 & -0.041 & -0.038 & 1.000 & & & & \\
\hline$N O N B I G \_N O N B I G(V 6)$ & -0.100 & -0.063 & -0.040 & -0.037 & -0.049 & 1.000 & & & \\
\hline$L A G \_R O \bar{A}(V 7)$ & 0.055 & 0.055 & -0.038 & -0.005 & 0.024 & -0.069 & 1.000 & & \\
\hline$L A G \_L O S S(V 8)$ & -0.055 & -0.045 & 0.033 & -0.002 & -0.019 & 0.048 & -0.706 & 1.000 & \\
\hline$L A G \_S I Z E(V 9)$ & 0.815 & 0.222 & -0.072 & -0.013 & 0.136 & -0.113 & 0.173 & -0.155 & 1.000 \\
\hline$L A G \_L E V(V 10)$ & 0.239 & 0.004 & -0.014 & 0.009 & 0.021 & 0.001 & -0.288 & 0.221 & 0.221 \\
\hline$L A G \_G R W(V 11)$ & -0.021 & 0.008 & -0.005 & 0.000 & -0.012 & 0.003 & 0.191 & -0.156 & -0.018 \\
\hline$L A G \quad E X P R A T I O(V 12)$ & 0.070 & 0.027 & -0.003 & -0.006 & -0.002 & -0.020 & 0.028 & 0.017 & 0.075 \\
\hline$L A G \_L I Q(V 13)$ & -0.209 & 0.007 & 0.024 & -0.011 & -0.022 & 0.017 & 0.119 & -0.072 & -0.215 \\
\hline$L A G \_I N V A R(V 4)$ & -0.168 & -0.079 & 0.013 & 0.021 & -0.043 & 0.007 & 0.091 & -0.092 & -0.168 \\
\hline$L A G_{-} M B(V 15)$ & 0.071 & 0.049 & 0.004 & 0.011 & 0.018 & 0.022 & -0.069 & 0.072 & -0.088 \\
\hline OWNER (V16) & -0.004 & 0.022 & -0.015 & 0.014 & 0.025 & -0.025 & 0.124 & -0.086 & 0.043 \\
\hline FOR $(V 17)$ & 0.436 & 0.189 & -0.053 & -0.016 & 0.083 & -0.077 & 0.184 & -0.149 & 0.456 \\
\hline$L A G \_O P I N O(V 18)$ & 0.000 & 0.011 & 0.009 & 0.008 & 0.002 & 0.002 & 0.027 & -0.028 & 0.013 \\
\hline
\end{tabular}

(Table 2, Panel B continued on next page) 
$\underline{\text { Panel B. Continued }}$

\begin{tabular}{|c|c|c|c|c|c|c|c|c|c|}
\hline & V10 & V11 & V12 & V13 & V14 & V15 & V16 & V17 & V18 \\
\hline$L A G \_L E V(V 10)$ & 1.000 & & & & & & & & \\
\hline$L A G \_G R W(V 11)$ & 0.041 & 1.000 & & & & & & & \\
\hline$L A G \_E X P R A T I O(V 12)$ & 0.049 & 0.008 & 1.000 & & & & & & \\
\hline$L A G \_L I Q(V 13)$ & -0.619 & -0.035 & -0.014 & 1.000 & & & & & \\
\hline$L A G \_I N V A R(V 4)$ & 0.188 & 0.079 & 0.029 & -0.144 & 1.000 & & & & \\
\hline$L A G M B(V 15)$ & 0.062 & 0.111 & 0.011 & 0.042 & -0.077 & 1.000 & & & \\
\hline OWNER (V16) & -0.020 & -0.013 & -0.056 & 0.035 & 0.016 & -0.059 & 1.000 & & \\
\hline FOR (V17) & -0.088 & 0.009 & 0.015 & 0.029 & -0.128 & 0.109 & 0.022 & 1.000 & \\
\hline LAG_OPINO (V18) & -0.022 & 0.004 & -0.006 & 0.009 & -0.005 & -0.006 & -0.010 & -0.006 & 1.000 \\
\hline
\end{tabular}

\section{Results for the Study Hypotheses}

Table 3 provides the results of Equation (1) to test hypothesis 1 . The non-audit services and initial audit have a significant negative association. However, the results reveal no significant relationship between non-audit services and the change from a large auditor to a small auditor. The result suggests that in the case of an initial audit where a large auditor is changed to a small auditor, non-audit services will likely be provided.

Table 3. Results of Hypothesis 1

\begin{tabular}{|c|c|c|c|c|}
\hline & Coefficient & p-value & Coefficient & p-value \\
\hline Intercept & $-6.6320^{* * *}$ & $(<.0001)$ & $-6.6560^{* * *}$ & $(<.0001)$ \\
\hline FIRST & $-0.2592^{* * *}$ & $(<.0001)$ & & \\
\hline$B I G N O N B I G$ & & & -0.0834 & $(0.5398)$ \\
\hline$N O \bar{N} B I G \quad B I G$ & & & $-0.3043^{* *}$ & $(0.0221)$ \\
\hline$B I G_{-} B I G$ & & & $-0.3184^{* * *}$ & $(0.0015)$ \\
\hline$N O N B I G N O N B I G$ & & & $-0.2624^{* *}$ & $(0.0362)$ \\
\hline$L A S T$ & $-0.2889^{* * *}$ & $(<.0001)$ & $-0.2895^{* * *}$ & $(<.0001)$ \\
\hline SIZE & $0.2120^{* * *}$ & $(<.0001)$ & $0.2122^{* * *}$ & $(<.0001)$ \\
\hline$L E V$ & $-0.4402^{* * *}$ & $(0.0005)$ & $-0.4380^{* * *}$ & $(0.0006)$ \\
\hline$G R W$ & -0.0006 & $(0.9926)$ & 0.0001 & $(0.9993)$ \\
\hline$M V$ & $0.1030^{* * *}$ & $(<.0001)$ & $0.1034^{* * *}$ & $(<.0001)$ \\
\hline$R O A$ & -0.2735 & $(0.2523)$ & -0.2770 & $(0.2465)$ \\
\hline $\mathrm{CFO}$ & $0.7791^{* * *}$ & $(0.0015)$ & $0.7818^{* * *}$ & $(0.0014)$ \\
\hline OWNER & 0.2303 & $(0.1390)$ & 0.2304 & $(0.1389)$ \\
\hline FORGI & $0.7320^{* * *}$ & $(0.0007)$ & $0.7322^{* * *}$ & $(0.0007)$ \\
\hline$B I G 4$ & $0.4997^{* * *}$ & $(<.0001)$ & $0.5184^{* * *}$ & $(<.0001)$ \\
\hline KOSPI & $0.1834^{* * *}$ & $(0.0009)$ & $0.1843^{* * *}$ & $(0.0009)$ \\
\hline IND & \multicolumn{2}{|c|}{ Included } & \multicolumn{2}{|c|}{ Included } \\
\hline YD & \multicolumn{2}{|c|}{ Included } & \multicolumn{2}{|c|}{ Included } \\
\hline Max-rescaled R2 & \multicolumn{2}{|c|}{0.1170} & \multicolumn{2}{|c|}{0.1173} \\
\hline Likelihood Ration & \multicolumn{2}{|c|}{$989.2028^{* * *}$} & \multicolumn{2}{|c|}{$991.32777^{* * *}$} \\
\hline$\#$ of $\mathrm{NASF}=1$ & \multicolumn{2}{|c|}{3,352} & \multicolumn{2}{|c|}{3,352} \\
\hline$\#$ of $\mathrm{NASF}=0$ & \multicolumn{2}{|c|}{8,226} & \multicolumn{2}{|c|}{8,226} \\
\hline Obs. & \multicolumn{2}{|c|}{11,578} & \multicolumn{2}{|c|}{11,578} \\
\hline
\end{tabular}

Table 4 reports the results of Equation (2) to test hypothesis 2. Consistent with hypothesis 2, we find that the coefficients on AUD_CH*InNASFEE are significantly negative for the third column, which shows Equation (2) by using AUD_CH as a proxy for BIG_NONBIG (-0.1571, $\mathrm{t}=-3.56)$. However, we find no significant relationship between $\ln \mathrm{AF}$ and AUD_CH2*InNASFEE, AUD_CH3*lnNASFEE and AUD_CH4*lnNASFEE. The result suggest that the initial audit fee discount in terms of non-audit services is probably offered in the case of changing to a small auditor from a large auditor. We continue to find evidence generally supporting our hypothesis 2 . 
Table 4. Results of Hypothesis 2

\begin{tabular}{|c|c|c|c|c|c|}
\hline & \multicolumn{5}{|c|}{ Proxy for AUD_CH } \\
\hline & FIRST & BIG_NONBIG & NONBIG_BIG & BIG_BIG & NONBIG_NONBIG \\
\hline & $\begin{array}{l}\text { Coefficient } \\
\text { (t-stat.) }\end{array}$ & $\begin{array}{c}\text { Coefficient } \\
\text { (t-stat.) }\end{array}$ & $\begin{array}{c}\text { Coefficient } \\
\text { (t-stat.) }\end{array}$ & $\begin{array}{l}\text { Coefficient } \\
\text { (t-stat.) }\end{array}$ & $\begin{array}{c}\text { Coefficient } \\
\text { (t-stat.) }\end{array}$ \\
\hline Intercept & $\begin{array}{c}8.6303^{* * *} \\
(82.68)\end{array}$ & $\begin{array}{c}8.6618^{* * *} \\
(83.06)\end{array}$ & $\begin{array}{c}8.6282^{* * *} \\
(82.52)\end{array}$ & $\begin{array}{c}8.6318^{* * *} \\
(82.31)\end{array}$ & $\begin{array}{c}8.6459^{* * *} \\
(82.53)\end{array}$ \\
\hline $\ln N A S F E E$ & $\begin{array}{c}0.0061^{* * *} \\
(12.98)\end{array}$ & $\begin{array}{c}0.0059^{* * *} \\
(13.55)\end{array}$ & $\begin{array}{c}0.0059^{* * *} \\
(13.31)\end{array}$ & $\begin{array}{c}0.0059^{* * *} \\
(13.15)\end{array}$ & $\begin{array}{c}0.0059^{* * *} \\
(13.26)\end{array}$ \\
\hline$A U D \_C H$ & $\begin{array}{c}-0.0467^{* * *} \\
(-4.38)\end{array}$ & $\begin{array}{c}-0.1159^{* * *} \\
(-5.67)\end{array}$ & $\begin{array}{c}-0.0412^{*} \\
(-1.78)\end{array}$ & $\begin{array}{c}0.0109 \\
(0.58)\end{array}$ & $\begin{array}{c}-0.0213 \\
(-1.26)\end{array}$ \\
\hline $\begin{array}{l}A U D \_C H^{*} \\
\ln N A \bar{S} F E E\end{array}$ & $\begin{array}{c}-0.0026^{* *} \\
(-2.14)\end{array}$ & $\begin{array}{c}-0.1571^{* * *} \\
(-3.56)\end{array}$ & $\begin{array}{c}-0.0501 \\
(-1.16)\end{array}$ & $\begin{array}{c}-0.0276 \\
(-0.87)\end{array}$ & $\begin{array}{c}-0.0551 \\
(-1.36)\end{array}$ \\
\hline$L A S T$ & $\begin{array}{c}0.0070 \\
(0.75)\end{array}$ & $\begin{array}{c}-0.2929^{* * * *} \\
(-6.97)\end{array}$ & $\begin{array}{c}-0.2893^{* * *} \\
(-6.86)\end{array}$ & $\begin{array}{c}-0.2882^{* * *} \\
(-6.83)\end{array}$ & $\begin{array}{c}-0.2898^{* * *} \\
(-6.87)\end{array}$ \\
\hline$L A G \_R O A$ & $\begin{array}{c}-0.2945^{* * *} \\
(-6.99)\end{array}$ & $\begin{array}{c}0.0204^{*} \\
(1.90)\end{array}$ & $\begin{array}{c}0.0201^{*} \\
(1.87)\end{array}$ & $\begin{array}{c}0.0201 * \\
(1.87)\end{array}$ & $\begin{array}{c}0.0200 * \\
(1.86)\end{array}$ \\
\hline$L A G_{-} L O S S$ & $\begin{array}{c}0.0200^{*} \\
(1.86)\end{array}$ & $\begin{array}{l}0.3665^{* * *} \\
(116.17)\end{array}$ & $\begin{array}{l}0.3676^{* * *} \\
(116.26)\end{array}$ & $\begin{array}{l}0.3675^{* * *} \\
(115.56)\end{array}$ & $\begin{array}{l}0.3670^{* * *} \\
(115.73)\end{array}$ \\
\hline$L A G \_L E V$ & $\begin{array}{c}0.14805^{* * *} \\
(6.24)\end{array}$ & $\begin{array}{c}-0.0175^{* *} \\
(-2.09)\end{array}$ & $\begin{array}{c}-0.0170^{* *} \\
(-2.04)\end{array}$ & $\begin{array}{c}-0.01684^{* *} \\
(-2.01)\end{array}$ & $\begin{array}{c}-0.0168^{* *} \\
(-2.01)\end{array}$ \\
\hline$L A G \_S I Z E$ & $\begin{array}{c}0.3677^{* * *} \\
(116.47)\end{array}$ & $\begin{array}{c}0.1471^{* * *} \\
(6.21)\end{array}$ & $\begin{array}{c}0.1468^{* * *} \\
(6.18)\end{array}$ & $\begin{array}{c}0.1481^{* * *} \\
(6.23)\end{array}$ & $\begin{array}{c}0.1487^{* * *} \\
(6.26)\end{array}$ \\
\hline$L A G \_G R W$ & $\begin{array}{c}-0.01782^{* *} \\
(-2.13)\end{array}$ & $\begin{array}{c}0.0081 \\
(0.68)\end{array}$ & $\begin{array}{c}0.00849 \\
(0.71)\end{array}$ & $\begin{array}{c}0.00847 \\
(0.71)\end{array}$ & $\begin{array}{c}0.0080 \\
(0.68)\end{array}$ \\
\hline$L A G \_E X P R A T I O$ & $\begin{array}{c}0.00742 \\
(0.62)\end{array}$ & $\begin{array}{c}-0.0054^{* * *} \\
(-3.84)\end{array}$ & $\begin{array}{c}-0.0055^{* * *} \\
(-3.95)\end{array}$ & $\begin{array}{c}-0.00553^{* * *} \\
(-3.91)\end{array}$ & $\begin{array}{c}-0.0054^{* * *} \\
(-3.87)\end{array}$ \\
\hline$L A G \_L I Q$ & $\begin{array}{c}-0.00546^{* * *} \\
(-3.86)\end{array}$ & $\begin{array}{c}0.0077 \\
(0.33)\end{array}$ & $\begin{array}{c}0.0065 \\
(0.28)\end{array}$ & $\begin{array}{c}0.00559 \\
(0.23)\end{array}$ & $\begin{array}{c}0.0049 \\
(0.21)\end{array}$ \\
\hline$L A G \_I N V A R$ & $\begin{array}{c}0.00519 \\
(0.22)\end{array}$ & $\begin{array}{c}0.0549^{* * *} \\
(18.66)\end{array}$ & $\begin{array}{c}0.0552^{* * *} \\
(18.69)\end{array}$ & $\begin{array}{c}0.05502^{* * *} \\
(18.62)\end{array}$ & $\begin{array}{c}0.0549^{* * *} \\
(18.59)\end{array}$ \\
\hline$L A G \_M B$ & $\begin{array}{c}0.05537^{* * *} \\
(18.77)\end{array}$ & $\begin{array}{c}-0.1041^{* * *} \\
(-4.44)\end{array}$ & $\begin{array}{c}-0.0999^{* * *} \\
(-4.25)\end{array}$ & $\begin{array}{c}-0.10108^{* * *} \\
(-4.3)\end{array}$ & $\begin{array}{c}-0.1022^{* * *} \\
(-4.35)\end{array}$ \\
\hline OWNER & $\begin{array}{c}-0.0993^{* * *} \\
(-4.23)\end{array}$ & $\begin{array}{c}0.3961^{* * *} \\
(11.61)\end{array}$ & $\begin{array}{c}0.3995^{* * *} \\
(11.67)\end{array}$ & $\begin{array}{c}0.40225^{* * *} \\
(11.75)\end{array}$ & $\begin{array}{c}0.4009^{* * *} \\
(11.72)\end{array}$ \\
\hline FORGI & $\begin{array}{c}0.39731^{* * *} \\
(11.63)\end{array}$ & $\begin{array}{c}-0.08344 \\
(-1.26)\end{array}$ & $\begin{array}{c}-0.08724 \\
(-1.32)\end{array}$ & $\begin{array}{c}-0.08792 \\
(-1.33)\end{array}$ & $\begin{array}{c}-0.08762 \\
(-1.32)\end{array}$ \\
\hline$L A G \_O P I N O$ & $\begin{array}{c}-0.08546 \\
(-1.29)\end{array}$ & $\begin{array}{c}-0.0933^{* * *} \\
(-5.74)\end{array}$ & $\begin{array}{c}-0.0862^{* * *} \\
(-5.29)\end{array}$ & $\begin{array}{c}-0.08714^{* * * *} \\
(-5.35)\end{array}$ & $\begin{array}{c}-0.0879^{* * *} \\
(-5.4)\end{array}$ \\
\hline $\begin{array}{l}\text { IND } \\
\text { YD }\end{array}$ & $\begin{array}{l}\text { Included } \\
\text { Included }\end{array}$ & $\begin{array}{l}\text { Included } \\
\text { Included }\end{array}$ & $\begin{array}{l}\text { Included } \\
\text { Included }\end{array}$ & $\begin{array}{l}\text { Included } \\
\text { Included }\end{array}$ & $\begin{array}{l}\text { Included } \\
\text { Included }\end{array}$ \\
\hline Adj. $\mathrm{R}^{2}$ & 0.7156 & 0.7164 & 0.7146 & 0.7144 & 0.7146 \\
\hline Obs. & 11578 & 11578 & 11578 & 11578 & 11578 \\
\hline
\end{tabular}

1) Notes: $*, * *, * * *$ represent significance at the 10,5 , and 1 percent levels, respectively.

\section{Additional Tests}

In this section, we conduct sensitivity tests to verify whether the above results are robust using the extended model. We present a regression analysis that incorporates the four audit change variables, BIG_NONBIG, NONBIG_BIG, BIG_BIG, and NONBIG_NONBIG, and the four interaction variables, $\ln N A S F E E^{*} \bar{B} I G \_N O N B I G, \ln N A S F E E^{*}$ $N O \overline{N B} B I_{-} B I G, \ln N A S F E \bar{E}^{*} B I G \_B I G$, and $\ln N A S F E E^{*} N O N B I G \_N O N B I G$.

The results are consistent with our hypotheses, and we find that the coefficients on lnNASFEE* BIG_NONBIG are significantly negative for the extended model. The results reveal no significant relationship between audit fees (lnAF) and $\ln N A S F E E^{*} N O N B I G_{-} B I G, \ln N A S F E E^{*} B I G_{-} B I G$, and $\ln N A S F E E^{*} N O N B I G \_N O N B I G$. However, audit fees have the significantly negative association with non-audit fees when changing from a large auditor to a small auditor. 
Table 5. Results of Hypothesis 2 by Using the Extended Model

\begin{tabular}{|c|c|c|}
\hline & Coefficient & (t-stat.) \\
\hline Intercept & $8.6748^{* * *}$ & $(82.87)$ \\
\hline $\ln N A S F E E$ & $0.0062^{* * *}$ & $(13.35)$ \\
\hline$B I G \quad N O N B I G$ & $-0.1206^{* * *}$ & $(-5.88)$ \\
\hline$N O \bar{N} B I G \_B I G$ & $-0.0497^{* *}$ & $(-2.15)$ \\
\hline$B I G \_B I G^{-}$ & 0.0032 & $(0.17)$ \\
\hline$N O \overline{N B} B I G \_N O N B I G$ & $-0.0312^{*}$ & $(-1.84)$ \\
\hline $\ln N A S F E E^{*} B I G \_N O N B I G$ & $-0.1608^{* * *}$ & $(-3.64)$ \\
\hline $\ln N A S F E E^{*} N O N B I G \_B I G$ & -0.0542 & $(-1.26)$ \\
\hline $\operatorname{lnNASFEE^{*}BIG\_ BIG-}$ & -0.0326 & $(-1.03)$ \\
\hline $\ln N A S F E E^{*} N O \bar{N} B I G \quad N O N B I G$ & -0.0592 & $(-1.47)$ \\
\hline$L A G \_R O A$ & $-0.2977^{* * *}$ & $(-7.08)$ \\
\hline$L A G_{-} L O S S$ & $0.0201^{*}$ & $(1.87)$ \\
\hline$L A G \_L E V$ & $0.1474^{* * *}$ & $(6.22)$ \\
\hline$L A G \_L E V$ & $0.3660^{* * *}$ & $(115.18)$ \\
\hline$L A G \_G R W$ & $-0.0180^{* *}$ & $(-2.16)$ \\
\hline$L A G \_E X P R A T I O$ & 0.0073 & $(0.61)$ \\
\hline$L A G_{-} L I Q$ & $-0.0054^{* * *}$ & $(-3.82)$ \\
\hline$L A G \_I N V A R$ & 0.0075 & $(0.32)$ \\
\hline$L A G \quad M B$ & $0.0552^{* * *}$ & $(18.73)$ \\
\hline$O W \bar{N} E R$ & $-0.1027^{* * *}$ & $(-4.38)$ \\
\hline FORGI & $0.3902^{* * *}$ & (11.44) \\
\hline LAG_OPINO & -0.0814 & $(-1.23)$ \\
\hline IND & \multicolumn{2}{|c|}{ Included } \\
\hline YD & \multicolumn{2}{|c|}{ Included } \\
\hline Adj. $\mathrm{R}^{2}$ & \multicolumn{2}{|c|}{0.7168} \\
\hline Obs. & \multicolumn{2}{|c|}{11578} \\
\hline
\end{tabular}

1) Notes: $*, * *, * * *$ represent significance at the 10,5 , and 1 percent levels, respectively.

2) See TABLE 1 for definition for other variables.

\section{SUMMARY AND CONCLUSION}

The purpose of this research is to verify whether non-audit services are provided without additional fees at the initial audit as a strategy to win an external audit contract, which could give the appearance of initial audit fee discount. The results are as follows.

Similar to Beck et al. (1988a), it is reconfirmed that non-audit services are generally not provided simultaneously in the case of an initial audit. However, if the type of auditor change is classified, and if it is changed from a Big 4 accounting firm to a non-Big 4 accounting firm, it tends to provide non-audit services at initial audit unlike other change types. This result suggests that a non-Big 4 accounting firm may use non-audit services in order to win external audit contracts. In order to verify this, entire samples are divided into four groups according to the types of auditor change and verified. As a result, non-audit services are provided at the initial audit, and the initial audit fee is appeared to be discounted accordingly, only when the independent auditor is changed from a Big 4 accounting firm to a nonBig 4 accounting firm. However, there is no meaningful relevance in any other types of changes. Therefore, if the auditor is changed from a Big 4 accounting firm to a non-Big 4 accounting firm, non-audit services are provided without additional fees in order to win an external audit contract, and the publication of audit fees with a division between the audit service fee and the non-audit service fee may give the appearance of a discounted audit service fee.

This research has scientific value in being the first investigation of the relevance between the initial audit fee discount and non-audit services according to the auditor change. Moreover, this research raises the issue regarding the actual nature of the non-audit services provided at initial audit. Since it should provide an audit service and non-audit service within the total fees similar to the audit service fee of the predecessor, the audit hours will be definitely decreased and, therefore, the quality of the audit will likely be degraded. Therefore, the accounting regulation agency needs to pay more attention to the type of auditor change and non-audit services which would be provided at the initial audit. And 
the description and nature of the non-audit services provided at the initial audit should be published to protect investors, and the adequacy of the total fees and audit and non-audit service fees needs to be reviewed more carefully.

\section{AUTHOR BIOGRAPHIES}

Hong-jo Park Ph.D., Associate Professor, Department of Accounting, Daegu University' Gyeongsanbuk-do, Republic of Korea. E-mail address: hjp@daugu.ac.kr (Main author)

Jeong-un Choi Ph.D., Assistant Professor, Department of Accounting, Daegu University' Gyeongsanbuk-do, Republic of Korea. E-mail address: jaychoi@daugu.ac.kr

Joonhei Cheung Ph.D., Assistant Professor, Department of Accounting, Daegu University' Gyeongsanbuk-do, Republic of Korea. E-mail address: janny81@daugu.ac.kr (Corresponding author)

\section{REFERENCES}

Antle, R., E. Gordon, G. Narayanamoorthy, and L. Zhou. 2006. The joint determination of audit fees, non-audit fees, and abnormal accruals, Review of Quantitative Finance and Accounting 27(3): 235-266.

Beck, P. J., T. J. Frecka, and I. Solomon. 1988a. A model of the market for MAS and audit services: Knowledge spillovers and auditorauditee bounding, Journal of Accounting Literature 7(1): 50-64.

Beck, P. J., T. J. Frecka, and I. Solomon. 1988b. An empirical analysis of the relationship between MAS involvement and auditor tenure: Implications for auditor independence, Journal of Accounting Literature 7(1): 65-84.

Becker, C. L., M. L. DeFond, J. Jiambalvo, and K. R. Subramanyam. 1998. The effect of audit quality on earnings management, Contemporary Accounting Research 15(1): 1-24.

Craswell, A. T., and J. R. Francis. 1999. "Pricing initial audit engagements: A test of competing theories, The Accounting Review 74(2): 201-216.

DeAngelo, L. E. 1981. Auditor size and audit quality, Journal of Accounting and Economics 3: 183-199.

Dee, C. C., A. A. Luiseged, and T. S. Nowlin. 2002. Earnings quality and auditor independence: An Examination using non-audit fee data, Working paper.

DeFond, M. L., K. Raghunandan, and K. R. Subramanyam. 2002. Do non-audit service fee impair auditor independence? Evidence from going concern audit opinions, Journal of Accounting Research 40(4): 1247-1274.

Desir, R., J. R. Casterella, and J. Kokina. 2014. "A reexamination of audit fees for initial audit engagements in the post-SOX period, Auditing: A Journal of Practice \& Theory 33(2): 59-78.

Dye, R. A. 1991. Informationally motivated auditor replacement, Journal of Accounting and Economics 14(4): 347-374.

Ettredge, M., and R. Greenberg. 1990. Determinants of fee cutting on initial audit engagements, Journal of Accounting Research 28(1): 198-210.

Francis, J. R., and D. T. Simon. 1987. A test of audit pricing in the small-client segment of the US audit market, The Accounting Review 62(1): 145-157.

Frankel, R. M., M. F. Johnson, and K. K. Nelson. 2002. The relation between auditors' fee for nonaudit services and earnings management, The Accounting Review 77(s-1): 71-105.

Ghosh, A., and S. Lustgarten. 2006. Pricing of initial audit engagements by large and small audit firms, Contemporary Accounting Research 23(2): 333-368.

Kim, B., J. Jang, and J. Cheung. The Effects of Non-audit Services on Audit Quality, Audit fee and Audit hour with Controlling for Endogeneity, Korean Management Review 45(2): 365-401.

Simon, D. T., and J. R. Francis. 1988. The effects of auditor change on audit fees: Tests of price cutting and price recovery, The Accounting Review 63(2): 255-269.

Simunic, D. A. 1984. Auditing, consulting, and auditor independence, Journal of Accounting Research 22(2): 679-702.

Walker, P. L., J. R. Casterella. 2000. The role of auditee profitability in pricing new audit engagements, Auditing: A Journal of Practice \& Theory 19(1): 157-167. 


\section{NOTES}

